\title{
Do different cone beam computed tomography exposure protocols influence subjective image quality prior to and after root canal treatment?
}

\author{
Andy Wai Kan Yeung ${ }^{1} \cdot$ Basak Harper $^{1} \cdot$ Chengfei Zhang $^{2} \cdot$ Prasanna Neelakantan $^{2} \cdot$ Michael M. Bornstein $^{1,3} \mathbb{C}$
}

Received: 19 November 2019 / Accepted: 11 August 2020 / Published online: 25 August 2020

(C) The Author(s) 2020

\begin{abstract}
Objectives The current study aimed to evaluate different CBCT exposure protocols and influencing factors affecting the subjective image quality of scans taken for endodontic indications.

Materials and methods Twelve extracted teeth, comprising of two sets of maxillary molars, premolars, canines and incisors, mandibular premolars, and molars, were endodontically treated, and either received a fiber or metal post. The teeth were scanned by CBCT imaging before and after root canal treatment, and after post insertion. Each scan was performed thrice, using an ultra low dose (ULD), standard (SM), and high-resolution mode (HR), respectively. Twelve observers - 4 endodontists, 4 periodontists, and 4 radiologistsassessed the subjective image quality using visual analogue scales (VAS). Potential influencing factors were evaluated including acquisition mode, observer specialty, stage of treatment, type of post, and type of tooth, using one-way ANOVA and $T$ test.

Results Teeth scanned with the ULD had the highest average VAS score (72.5), followed by HR (70.2), and SM (69.0) for values pooled from all teeth and observers. CBCT acquisition mode was not a significant influencing factor on the VAS scores. Observer specialty, stage of treatment, type of post, and type of tooth were significant influencing factors.

Conclusions Based on the present in vitro data, a low-dose CBCT mode seems not to negatively affect the perception of image quality. Clinical relevance The findings from this in vitro study demonstrate that a low-dose CBCT mode might have potential for diagnostics prior to or following endodontic treatment.
\end{abstract}

Keywords Cone beam computed tomography $\cdot$ Dose optimization $\cdot$ Root canal treatment $\cdot$ Image quality $\cdot$ Low dose

\section{Introduction}

The pitfalls of two-dimensional (2D) radiographic imaging in endodontics are well known [1]. For diagnostic purposes, conventional radiographs may have limitations, such as to identify all root canals including blocked canals, evaluate the morphology of tooth and root canal systems, localize broken instruments, and assess the relationship between the roots and

Michael M. Bornstein

michael.bornstein@unibas.ch

1 Oral and Maxillofacial Radiology, Applied Oral Sciences and Community Dental Care, Faculty of Dentistry, The University of Hong Kong, Hong Kong SAR, China

2 Endodontology, Faculty of Dentistry, The University of Hong Kong, Hong Kong SAR, China

3 Department of Oral Health \& Medicine, University Center for Dental Medicine Basel UZB, University of Basel, Basel, Switzerland the maxillary sinus or the mandibular canal [2]. Detection of cracks/fractures is also challenging using conventional radiographs, when the fracture lines are along the direction of the radiation beam [3]. If available, a surgical microscope can be helpful [4]. Three-dimensional (3D) imaging techniques such as cone beam computed tomography (CBCT) can help offset many of these problems. Nevertheless, there is considerable ambiguity in offering a decisive suggestion to routinely use $\mathrm{CBCT}$ for diagnostic procedures in endodontics. This is mainly related to the higher radiation dose of $\mathrm{CBCT}$ relative to conventional radiography $[5,6]$.

Contemporary root canal treatment demonstrates a steady shift toward conservation of tooth structure, and minimally invasive access cavity designs are being increasingly suggested [7]. With less direct intraoral visualization, the relevance of radiographic diagnostic imaging to offer more information regarding the root canal anatomy of a tooth prior to root canal treatment is becoming more important especially if a surgical microscope is not available [8]. However, the 
radiation dose of $\mathrm{CBCT}$, despite being lower than that of conventional CT, negates routine clinical usage, when one applies the principles of as low as reasonable achievable (ALARA) and as low as diagnostically acceptable (ALADA) [9]. Therefore, CBCT is still considered as an adjunctive imaging modality for endodontic purposes $[5,6,10]$.

CBCT devices usually allow clinicians to scan with different protocols, such as high resolution, standard, and even lowdose modes [11]. To achieve dose optimization, clinicians should consider using low-dose protocols for CBCT scans when possible [12]. For instance, an established low-dose protocol for pediatric CBCT may reduce as much as $50 \%$ of radiation dose compared to the standard exposure as recommended by the manufacturer [13]. CBCT is recommended as an efficient method of studying root canal systems [14], and high-resolution settings have been recommended by the literature and manufacturers for assessment of root canal anatomy [15]. Thus, there is a need to investigate standard and lowdose protocols, based on the fact that the three imaging protocols - high resolution, standard, and low dose- have not yet been assessed and compared for their impact on subjective image quality to evaluate root canal systems prior to or after root canal treatment.

Therefore, the goal of the present study was to evaluate different $\mathrm{CBCT}$ exposure protocols and potential influencing factors on the subjective image quality of CBCT scans taken for endodontic indications. The study was designed as an in vitro investigation using a cohort of mandibular and maxillary teeth that were scanned by CBCT before root canal treatment, after root canal treatment, and after post placement. The scans used one standard, one high-resolution, and one custom low-dose protocol, respectively. The primary aim was to evaluate, if observers gave significantly different ratings to images acquired by different CBCT settings. Secondary aims included the assessment of the influence of observer specialty, treatment stage, type of post, and tooth type on the ratings for subjective image quality.

\section{Materials and methods}

\section{Tooth selection}

All extracted teeth used in this study were collected from an existing pool of teeth. The study protocol was approved by the Institutional Review Board of the university (UW 17-206). Single-rooted and multi-rooted teeth that were included for this study were divided into two groups (Fig. 1). Each group comprised of six teeth including one for each of the following: maxillary incisor, maxillary canine, maxillary premolar, maxillary molar, mandibular premolar, and mandibular molar. Premolars and molars from the maxilla and mandible were included due to their heterogenous root canal morphology.

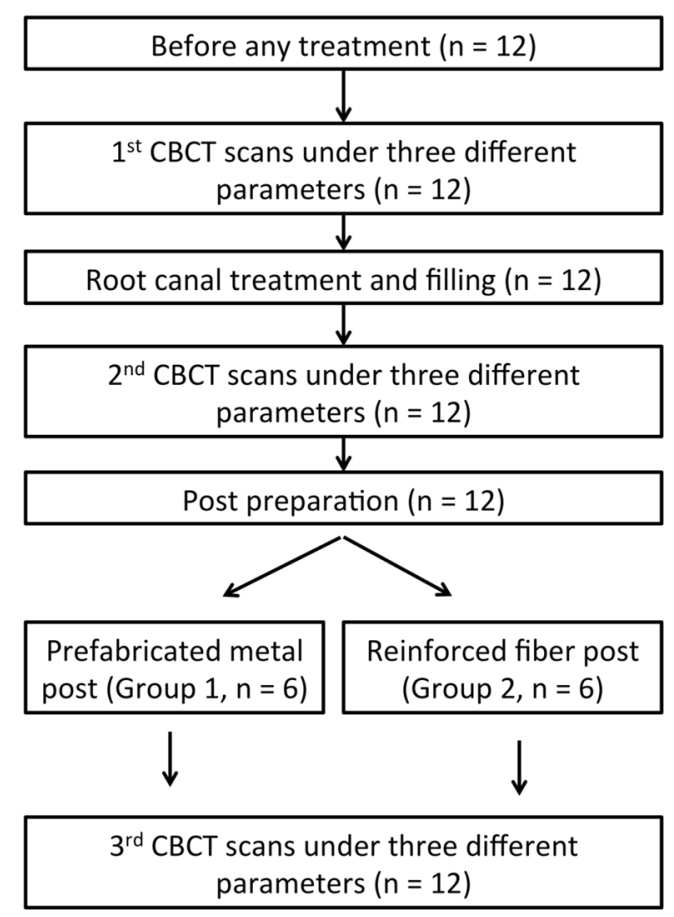

Fig. 1 Flow-chart illustrating the workflow for the 12 teeth processed and analyzed

The first group of teeth received root canal treatment with a fiber-reinforced composite post, whereas the second group of teeth received root canal treatment with a prefabricated metal post. Thus, for the two study groups tested (i.e., fiberreinforced composite post against metal post), this resulted in a total of 12 teeth to be included.

\section{Pre-operative CBCT scanning}

According to the method described by Shelley et al. [16], six teeth from each group were mounted in a customized silicone mold simulating soft tissues (Fig. 2) for CBCT scans with the ProMax 3D Mid device (Planmeca Oy, Helsinki, Finland) using three different protocols:

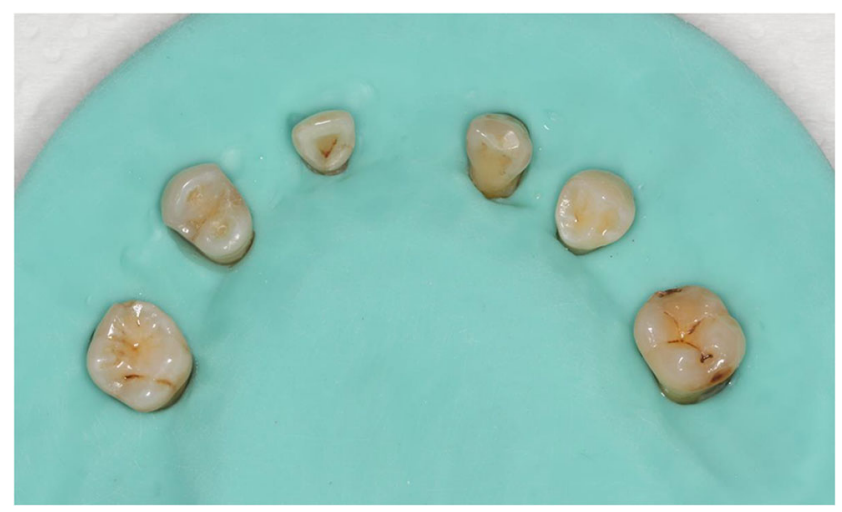

Fig. 2 Representative image of the six teeth from one group mounted in a customized silicone mold simulating soft tissues prior to root canal treatment 
1) High-resolution mode (HR): $90 \mathrm{kV}, 10 \mathrm{~mA}$, exposure time $15 \mathrm{~s}$, field of view (FOV) of $8 \times 5 \mathrm{~cm}$, voxel size of $0.15 \mathrm{~mm}$

2) Standard mode (SM; default setting given by the manufacturer): $90 \mathrm{kV}, 8 \mathrm{~mA}$, exposure time $12 \mathrm{~s}, \mathrm{FOV}$ of $8 \times 5$ $\mathrm{cm}$, voxel size of $0.2 \mathrm{~mm}$

3) Ultra low dose mode (ULD; as provided by the manufacturer): $90 \mathrm{kV}, 5.6 \mathrm{~mA}$, exposure time $4 \mathrm{~s}$, FOV of $8 \times 5$ $\mathrm{cm}$, voxel size of $0.2 \mathrm{~mm}$

\section{Root canal treatment}

Root canals of all 12 teeth were prepared with Wave One Gold instruments "Medium" size (Dentsply Sirona Endodontics, York, PA, USA) using $5 \mathrm{~mL}$ of $2 \%$ sodium hypochlorite as the root canal irrigant. Following completion of root canal instrumentation, the root canals were flushed with sterile saline and dried with absorbent paper points (Dentsply Sirona Endodontics, York, PA, USA). The root canals were filled using gutta-percha cones and accessory cones, with an epoxy resin-based root canal sealer (AH Plus, Dentsply DeTrey, Konstanz, Germany) using cold lateral compaction technique.

Following root canal treatment, all teeth were scanned using $\mathrm{CBCT}$ as mentioned previously, using 3 protocols.

\section{Endodontic post placement}

For the prefabricated metal post (group 1), the root canal fillings of the teeth were removed after root canal treatment to leave behind a $5 \mathrm{~mm}$ plug of apical gutta-percha. Following this, red $(1.25 \mathrm{~mm})$, black $(1.5 \mathrm{~mm})$, and yellow $(1 \mathrm{~mm})$ stainless steel posts (ParaPost ${ }^{\circledR}$ XPTM Stainless Steel Post, Coltene/ Whaledent Inc., Cuyahoga Falls, OH, USA) were cemented in the root canals of anteriors, premolars, and molars respectively, using resin cement (RelyX ${ }^{\mathrm{TM}}$ Unicem cement, 3M ESPE, St. Paul, MN, USA). The teeth were then filled with composite resin material (Filtex Z100, 3M ESPE, St. Paul, MN, USA).

For the fiber-reinforced composite post group (group 2), the root canal fillings of the teeth were removed after root canal treatment to leave behind a $5 \mathrm{~mm}$ plug of apical guttapercha. Afterwards, $1.6 \mathrm{~mm}$ (molars) and $1.9 \mathrm{~mm}$ (anteriors and premolars) fiber posts (RelyX ${ }^{\mathrm{TM}}$ Fiber Post, $3 \mathrm{M}$ ESPE, St. Paul, MN, USA) were luted with resin cement (RelyX ${ }^{\mathrm{TM}}$ Unicem cement). The teeth were then filled with composite resin material (Filtex Z100).

Following placement of the endodontic posts, all teeth were scanned using CBCT as mentioned previously, using 3 protocols.
Image quality evaluation of the $\mathrm{CBCT}$ scans

The CBCT images were evaluated by 12 observers form different specialties (4 from endodontology, 4 from periodontology, and 4 radiologists; all had at least 3 years of postgraduate training that involved $\mathrm{CBCT}$ analysis). All observers had a total of $18 \mathrm{CBCT}$ sets to analyze from 2 groups of teeth using 3 different CBCT settings (HR, SM, and ULD) at 3 different treatment stages (before root canal treatment, after root canal treatment, and after post insertion). The observers graded all scans on a visual analogue scale (VAS, 0-100) for each tooth separately assessing the subjective image quality. The observers were all instructed to focus on the sufficiency of image quality for various endodontic diagnoses, such as the evaluation of root canal filling and placement of the posts. The scans were presented to the observers unlabeled, and in a randomized sequence. The observers could modify the scans including brightness and contrast. They all evaluated the images on the same computer monitor (22-inch LED, $1920 \times 1080$ pixels, model $223 \mathrm{~V}$, Philips) in a darkened room.

\section{Statistical analysis}

Descriptive statistics were evaluated first. Inter-observer reliability was assessed using intra-class correlation coefficients (ICC) [17]. Then, the VAS scores were compared to reveal if images acquired by ULD, SM, and HR modes had any differences in the ratings of subjective image quality. Afterwards, it was assessed if observer specialty, stage of treatment, type of post, and type of tooth were influencing factors on the VAS scores. All these factors, except type of post, were tested by one-way ANOVA. The effect of the type of post on VAS scores was tested by independent $t$ test. Bonferroni correction was considered to adjust for multiple testing.

The significance level was set at $p=0.01$. All analyses were performed in SPSS (Version 25.0, IBM Corp., Armonk, New York, USA).

\section{Results}

Representative CBCT images taken with the three exposure settings before root canal treatment, after root canal treatment, with metal post inserted, and with fiber post inserted are presented in Fig. 3.

\section{VAS scores for the three CBCT settings}

Overall, teeth scanned with ULD had the highest average VAS score (72.5), followed by HR (70.2), and then SM mode (69.0) for values pooled from all teeth and all observers (Table 1). Periodontists gave the highest average VAS score (72.7, SD 17.6), followed by radiologists (70.8, SD 15.7), and 


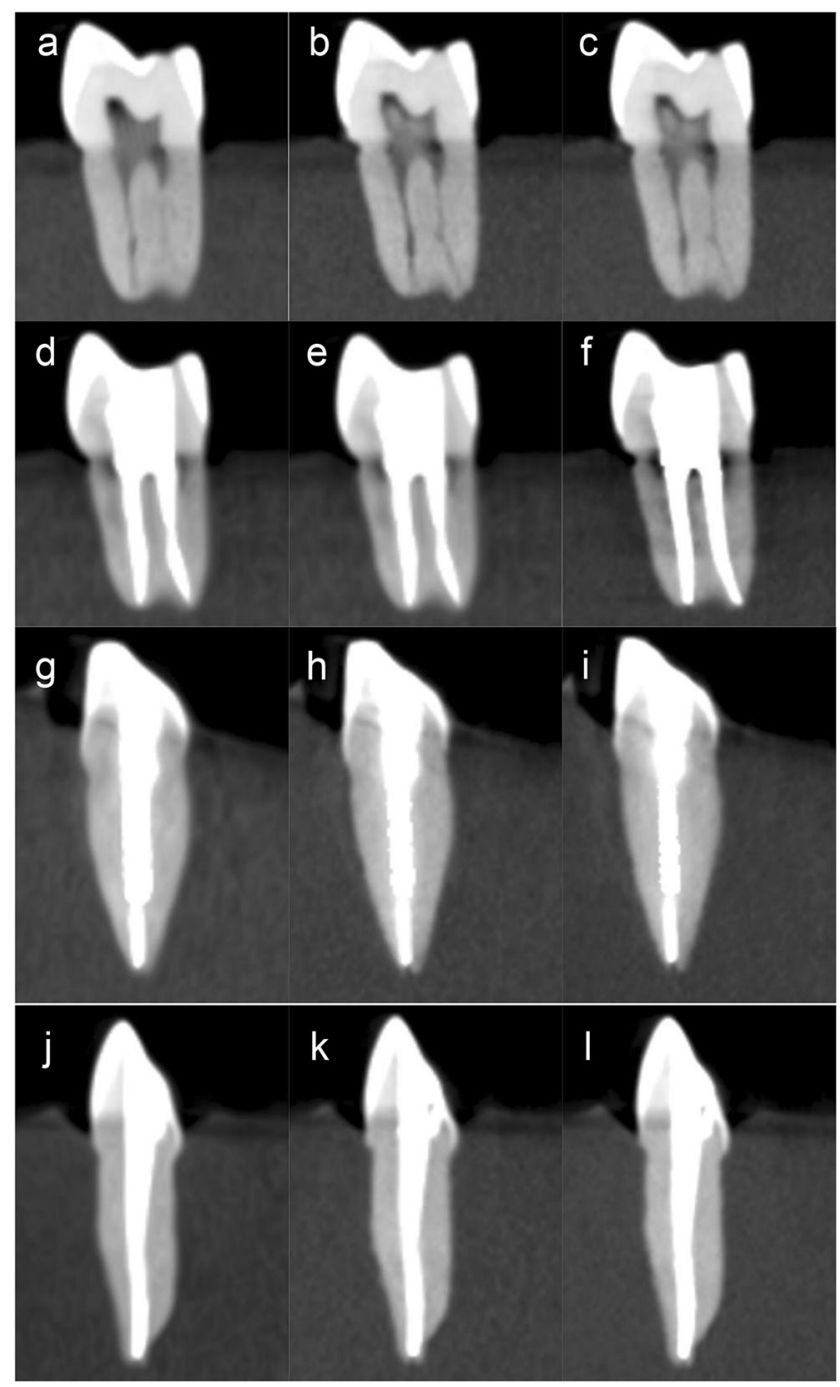

Fig. 3 Representative CBCT images of a mandibular premolar before root canal treatment with a ultra low dose mode (ULD), b standard mode (SM), and $\mathbf{c}$ high-resolution mode (HR), and after root canal treatment with $\mathbf{d}$ ULD, e SM, and $\mathbf{f}$ HR. Furthermore, a maxillary canine is depicted with an inserted metal post using $\mathbf{g}$ ULD, $\mathbf{h} \mathrm{SM}$, and $\mathbf{i} \mathrm{HR}$, and with a fiber post using $\mathbf{j}$ ULD, $\mathbf{k}$ SM, and $\mathbf{I} \mathrm{HR}$

endodontists (68.2, SD 20.4). With regards to the sequence of treatment, teeth scanned before root canal treatment had the highest rating (82.4, SD 11.8) compared to after root canal treatment (61.7, SD 17.4), and after post insertion (67.6, SD 17.6). Teeth with a fiber post (73.4, SD 14.8) exhibited higher ratings than those with a metal post (61.7, SD 18.5). In terms of type of tooth, maxillary canines had the highest rating (75.8, SD 17.5), followed by maxillary incisors (74.8, SD 17.9), and the molars (maxilla: 66.7, SD 23.0; mandible: 64.0, SD 24.6; Table 2).

\section{Inter-observer reliability}

ICC values showing inter-observer reliability are listed in Table 3. Overall, the values ranged from poor to good.
Endodontists and radiologists tended to have higher ICC values than periodontists. The number of ICC values (out of a total of 15) demonstrating moderate to good agreement (> 0.50 ) for endodontist, radiologist, and periodontist groups were 9,7 , and 5 respectively.

\section{Significance of potential influencing factors on VAS scores}

Statistical analyses demonstrated that the CBCT acquisition mode was not a significant influencing factor on the VAS scores $(p=0.048)$ for subjective image quality (Table 4$)$. Meanwhile, all other factors evaluated were significant influencing factors. Regarding observer specialty, periodontists gave generally higher mean VAS scores (on average 4.5 points higher) than endodontists $(p=0.008)$. Regarding stage of treatment, mean VAS score for teeth before root canal treatment was 14.8 points higher than after post insertion, which was in turn 5.9 points higher than after root canal treatment $(p=0.001)$. Teeth with fiber posts inserted had a mean VAS score that was 5.0 points higher than teeth with metal posts inserted $(p<0.001)$. With regard to the type of tooth, mean VAS scores of maxillary canines and maxillary incisors were significantly higher than those of maxillary premolars and mandibular premolars, which in turn were significantly higher than those of maxillary molars and mandibular molars $(p<0.001)$.

\section{Discussion}

The primary aim of the current study was to evaluate, if observers gave significantly different ratings to CBCT image quality acquired by different exposure settings. Secondary aims were to assess the influence of observer specialty, treatment stage, type of post, and tooth type on VAS ratings. The major findings were that $\mathrm{CBCT}$ images acquired by different settings did not result in significantly different ratings. On the contrary, it was found that rather the other factors like observer specialty, treatment stage, type of post, and tooth type were influencing the VAS values, and thus, the subjective perception of image quality significantly.

The present findings are relatively novel, as high-resolution CBCT images have been often recommended and reported to be superior to low-dose and standard modes for endodontic indications, such as to assess root fractures with higher accuracy $[18,19]$. However, the use of high-resolution CBCT imaging has been reported to result in higher radiation doses specifically to the eye lens and thyroid gland [20]. Indeed, the use of low-resolution CBCT imaging has been recommended for evaluating endodontic surgery with retrograde root filling [21]. However, that recommendation was based on the calculation of contrast-to-noise (CNR) ratio that involved no 
Table 1 Overview of the subjective image quality of the CBCT scans for each type of tooth for all three imaging modalities from all observers (VAS scores)

\begin{tabular}{|c|c|c|c|c|c|c|c|}
\hline & \multicolumn{4}{|l|}{ Maxilla } & \multicolumn{2}{|l|}{ Mandible } & \multirow[t]{2}{*}{ Average } \\
\hline & Incisor & Canine & Premolar & Molar & Premolar & Molar & \\
\hline \multicolumn{8}{|l|}{ Overall } \\
\hline Ultra low dose mode & $76.7(15.8)$ & $77.8(14.9)$ & $72.1(19.3)$ & $71.3(18.3)$ & $72.1(19.4)$ & $65.2(23.5)$ & $72.5(15.6)$ \\
\hline Standard mode & $73.9(18.6)$ & $72.4(20.5)$ & $69.6(21.5)$ & $62.2(25.1)$ & $72.4(19.6)$ & $63.8(25.1)$ & $69.0(18.8)$ \\
\hline High-resolution mode & $73.8(19.3)$ & $77.2(16.5)$ & $68.6(24.2)$ & $66.7(24.5)$ & $71.7(20.2)$ & $63.0(25.4)$ & $70.2(19.5)$ \\
\hline \multicolumn{8}{|l|}{ Before root canal treatment } \\
\hline Ultra low dose mode & $79.0(14.7)$ & $80.4(14.6)$ & $78.0(16.2)$ & $76.9(15.8)$ & $78.6(14.2)$ & $75.0(20.1)$ & $78.0(13.5)$ \\
\hline Standard mode & $85.7(9.4)$ & $85.5(8.2)$ & $84.9(10.0)$ & $83.2(10.2)$ & $85.5(8.8)$ & $84.1(9.5)$ & $84.8(8.6)$ \\
\hline High-resolution mode & $85.5(12.8)$ & $85.7(12.2)$ & $85.8(11.6)$ & $84.0(13.4)$ & $84.1(12.8)$ & $82.1(11.8)$ & 84.5 (11.9) \\
\hline \multicolumn{8}{|l|}{ After root canal treatment } \\
\hline Ultra low dose mode & $72.0(17.3)$ & $73.8(15.9)$ & $65.3(20.0)$ & $66.3(17.8)$ & $62.1(20.9)$ & $57.6(22.4)$ & $66.2(15.3)$ \\
\hline Standard mode & $67.5(17.7)$ & $67.6(17.6)$ & $61.1(20.5)$ & $52.3(23.6)$ & $60.1(20.2)$ & $52.9(24.0)$ & $60.2(16.9)$ \\
\hline High-resolution mode & $65.5(18.7)$ & $67.5(17.7)$ & $54.5(23.2)$ & $54.2(25.2)$ & $59.0(22.1)$ & $51.6(25.0)$ & $58.7(19.6)$ \\
\hline \multicolumn{8}{|l|}{ After metal post } \\
\hline Ultra low dose mode & $78.6(14.8)$ & $78.2(13.9)$ & $72.8(19.7)$ & $68.7(22.1)$ & $74.4(20.5)$ & $63.2(25.5)$ & $72.6(18.6)$ \\
\hline Standard mode & $62.4(16.2)$ & $54.8(23.8)$ & $50.3(25.7)$ & $42.2(20.2)$ & $60.8(19.8)$ & $43.2(23.5)$ & $52.3(15.5)$ \\
\hline High-resolution mode & $68.8(14.6)$ & $73.9(15.4)$ & $58.1(25.3)$ & $50.3(23.9)$ & $65.5(18.2)$ & $46.1(25.5)$ & $60.4(16.5)$ \\
\hline \multicolumn{8}{|l|}{ After fiber post } \\
\hline Ultra low dose mode & $79.4(15.6)$ & $80.4(14.4)$ & $73.0(21.3)$ & $72.4(18.4)$ & $77.0(17.9)$ & $63.0(25.7)$ & $74.2(14.3)$ \\
\hline Standard mode & $74.4(24.4)$ & $73.5(24.2)$ & $75.5(11.5)$ & $59.9(25.4)$ & $82.3(10.1)$ & $65.9(22.2)$ & $71.9(16.2)$ \\
\hline High-resolution mode & $72.3(25.4)$ & $82.8(12.2)$ & $72.8(22.7)$ & $73.8(16.1)$ & $78.6(12.7)$ & $64.3(24.1)$ & $74.1(14.9)$ \\
\hline
\end{tabular}

The VAS score ranged 0-100, with 100 being "perfect" for diagnostic purposes

$\mathrm{SD}$ values are in brackets

Table 2 Overview of subjective image quality of the CBCT scans for each type of tooth for all three specialties of observers (VAS scores)

\begin{tabular}{|c|c|c|c|c|c|c|c|}
\hline & \multicolumn{4}{|l|}{ Maxilla } & \multicolumn{2}{|l|}{ Mandible } & \multirow[t]{2}{*}{ Average } \\
\hline & Incisor & Canine & Premolar & Molar & Premolar & Molar & \\
\hline \multicolumn{8}{|l|}{ Endo } \\
\hline Before root canal treatment & $90.4(6.3)$ & $90.6(6.2)$ & $88.3(8.8)$ & $85.5(10.8)$ & $88.3(8.5)$ & $83.5(18.7)$ & $87.7(7.0)$ \\
\hline After root canal treatment & $67.0(22.8)$ & $68.3(21.7)$ & $54.4(26.9)$ & $51.0(28.1)$ & $61.0(23.7)$ & $52.0(28.7)$ & $59.0(19.6)$ \\
\hline After metal post insertion & $66.8(18.1)$ & $61.9(23.6)$ & $53.5(28.7)$ & $39.0(24.1)$ & $60.3(21.5)$ & $34.3(25.5)$ & $52.6(15.1)$ \\
\hline After fiber post insertion & $64.1(31.9)$ & $72.2(26.1)$ & $61.3(25.2)$ & $58.8(26.6)$ & $78.3(14.2)$ & $45.3(27.0)$ & $63.3(15.7)$ \\
\hline \multicolumn{8}{|l|}{ Perio } \\
\hline Before root canal treatment & $82.0(13.2)$ & $82.4(12.3)$ & $82.1(12.0)$ & $82.5(10.1)$ & $80.5(14.6)$ & $80.6(12.6)$ & $81.7(12.0)$ \\
\hline After root canal treatment & $68.5(17.0)$ & $69.8(15.8)$ & $64.4(17.7)$ & $62.5(20.5)$ & $61.9(20.9)$ & $59.3(20.1)$ & $64.4(17.7)$ \\
\hline After metal post insertion & $70.4(18.5)$ & $70.5(23.5)$ & $61.3(26.2)$ & $57.8(24.2)$ & $67.3(22.4)$ & $60.7(24.2)$ & $64.7(22.1)$ \\
\hline After fiber post insertion & $81.3(9.0)$ & $83.6(7.8)$ & $81.7(9.0)$ & $73.8(19.9)$ & $80.1(10.5)$ & $75.7(12.6)$ & $79.3(10.4)$ \\
\hline \multicolumn{8}{|l|}{ Radiology } \\
\hline Before root canal treatment & $77.8(14.0)$ & $78.6(13.4)$ & $78.3(16.2)$ & $76.0(17.3)$ & $79.5(11.6)$ & $77.1(12.2)$ & $77.9(13.6)$ \\
\hline After root canal treatment & $69.5(13.1)$ & $70.7(13.4)$ & $62.2(18.1)$ & $59.3(18.7)$ & $58.3(18.3)$ & $50.8(21.3)$ & $61.8(14.9)$ \\
\hline After metal post insertion & $72.7(12.1)$ & $74.5(11.7)$ & $66.3(19.3)$ & $64.3(18.0)$ & $73.0(14.0)$ & $57.5(19.3)$ & $68.1(14.9)$ \\
\hline After fiber post insertion & $80.8(14.3)$ & $80.9(13.1)$ & $78.3(11.4)$ & $73.6(10.3)$ & $79.6(17.0)$ & $72.2(16.0)$ & $77.6(13.0)$ \\
\hline
\end{tabular}

The VAS score ranged 0-100, with 100 being "perfect" for diagnostic purposes

$\mathrm{SD}$ values are in brackets 
Table 3 Inter-observer reliability of VAS scores amongst different groups of specialty with different acquisition modes at each stage of treatment

\begin{tabular}{lcccc}
\hline & Overall & Endo & Perio & Radiology \\
\hline Overall & & & & \\
Ultra low dose mode & 0.607 & 0.583 & 0.151 & 0.237 \\
Standard mode & 0.870 & 0.698 & 0.572 & 0.795 \\
High-resolution mode & 0.837 & 0.698 & 0.564 & 0.674 \\
Before root canal treatment & & & & \\
Ultra low dose mode & 0.063 & 0.216 & 0.222 & 0.339 \\
Standard mode & 0.037 & 0.224 & 0.143 & 0.348 \\
High-resolution mode & 0.037 & 0.127 & 0.074 & 0.563 \\
After root canal treatment & & & & \\
Ultra low dose mode & 0.663 & 0.574 & 0.173 & 0.492 \\
Standard mode & 0.549 & 0.284 & 0.119 & 0.436 \\
High-resolution mode & 0.428 & 0.107 & 0.274 & 0.063 \\
After metal post & & & & \\
Ultra low dose mode & 0.745 & 0.680 & 0.098 & 0.581 \\
Standard mode & 0.899 & 0.743 & 0.561 & 0.866 \\
High-resolution mode & 0.888 & 0.753 & 0.524 & 0.871 \\
After fiber post & & & & \\
Ultra low dose mode & 0.316 & 0.299 & 0.187 & 0.181 \\
Standard mode & 0.775 & 0.619 & 0.487 & 0.639 \\
High-resolution mode & 0.751 & 0.640 & 0.567 & 0.394 \\
\hline
\end{tabular}

Intraclass correlation coefficient (ICC)

$<0.50=$ poor, $0.50-0.75=$ moderate, $0.75-0.90=$ good,$>0.90=$ excel lent [17] clinical judgment. The present evaluation was based on subjective perception of the image quality by different specialists in dental medicine, whereas previous studies either focused on observers' accuracy on detecting lesions on CBCT images, or the more mechanistic CNR value that involves no direct observer judgment. Regarding the assessment of root canal morphology, previous studies have demonstrated the usefulness of CBCT imaging for this evaluation in both deciduous and permanent teeth, without investigating into the optimal CBCT setting for this purpose [14, 22]. Results from the present study imply that CBCT scans with a low-dose protocol may be adequate for evaluation of root canal systems.

There are no reports on the effect of different observer specialties on the subjective image quality perception of CBCT images. A recent study reported that periodontists are more likely to change their treatment plan and suggest extraction of teeth when CBCT images were provided to them to assess moderate to difficult endodontic cases, a finding that was contrasting outcomes from endodontists [23]. For nonCBCT images, it has been shown that endodontists demonstrated better inter-observer agreement on diagnosing periapical lesions with periapical radiographs [24]. Meanwhile, observer specialty was not a significant influencing factor on the resulting subjective image quality assessment when pathologists, oral medicine specialists, radiologists, and oral surgeons used panoramic images to diagnose oral unilocular radiolucent lesions [25]. Nevertheless, the results of the present study suggest that endodontists may be more consistent in radiographic assessments for evaluation of root canal systems prior to and following treatment using 3D imaging
Table 4 Analysis of potential influencing factors on the subjective image quality of $\mathrm{CBCT}$ images

\begin{tabular}{|c|c|c|c|}
\hline Influencing factor & & Statistical test & $P$ value \\
\hline \multirow[t]{2}{*}{ Acquisition mode } & $\begin{array}{l}\text { (1) } \mathrm{ULD}^{\mathrm{a}} \\
\text { (2) } \mathrm{SM}^{\mathrm{a}}\end{array}$ & \multirow[t]{2}{*}{ One-way ANOVA } & \multirow[t]{2}{*}{0.048} \\
\hline & (3) $\mathrm{HR}^{\mathrm{a}}$ & & \\
\hline \multirow[t]{2}{*}{ Observer specialty } & $\begin{array}{l}\text { (1) Endodontology }{ }^{a} \\
\text { (2) Radiology }\end{array}$ & \multirow[t]{2}{*}{ One-way ANOVA } & \multirow[t]{2}{*}{0.008} \\
\hline & (3) Periodontology ${ }^{\mathrm{b}}$ & & \\
\hline \multirow[t]{2}{*}{ Stage of treatment } & $\begin{array}{l}\text { (1) Before root canal treatment } \mathrm{t}^{\mathrm{a}} \\
\text { (2) After root canal treatment } \mathrm{b}^{\mathrm{b}}\end{array}$ & \multirow[t]{2}{*}{ One-way ANOVA } & \multirow[t]{2}{*}{0.001} \\
\hline & (3) After post insertion ${ }^{c}$ & & \\
\hline Type of post & $\begin{array}{l}\text { (1) } \text { Metal }^{\mathrm{a}} \\
\text { (2) } \text { Fiber }^{\mathrm{b}}\end{array}$ & $T$ test & $<0.001$ \\
\hline \multirow[t]{5}{*}{ Type of tooth } & $\begin{array}{l}\text { (1) Upper molar }{ }^{\mathrm{a}} \\
\text { (2) Upper premolar }\end{array}$ & \multirow[t]{5}{*}{ One-way ANOVA } & \multirow[t]{5}{*}{$<0.001$} \\
\hline & (3) Upper canine ${ }^{c}$ & & \\
\hline & (4) Upper incisor ${ }^{\mathrm{c}}$ & & \\
\hline & (5) Lower premolar ${ }^{\mathrm{b}}$ & & \\
\hline & (6) Lower molar ${ }^{\mathrm{a}}$ & & \\
\hline
\end{tabular}

Groups identified by different superscripts were significantly different at $p<0.01^{*}$

* Bonferroni correction $=$ significance level/the number of hypotheses $(0.05 / 5)$ 
techniques. Furthermore, as periodontists gave generally higher mean VAS scores in the present study, it might be suggested that radiologists and endodontists seem to be more critical concerning CBCT image quality for the evaluation of endodontic indications.

The observer ratings for $\mathrm{CBCT}$ images of the teeth after fiber post insertion were generally higher than those after root canal treatment prior to post insertion. These findings are not totally clear, but it may be speculated that the observers tended to focus on evaluating the root filling materials, such as their homogeneity and length, when they rated the images without posts inserted. Thus, they may have also assessed somewhat the quality of the root canal filling itself rather than the actual image quality. A recent ex vivo study reported that root-filled teeth resulted in CBCT images of inferior quality to detect apical periodontitis compared to non-root-filled counterparts [26]. CBCT imaging has also been said to be inferior to periapical images produced by F-speed films and phosphor plates to evaluate homogeneity and length of root fillings [27]. For posts, especially metallic ones, inserted after conventional root canal treatment, the impact of scatter and noise on the subjective image quality has to be taken into serious account [28], which makes it even more important to select the imaging modality with the best potential, but lowest radiation dose exposure. In the current study, teeth after metal post insertion, but not fiber post, had the lowest mean VAS scores, compared to those before root canal treatment and after root canal treatment without post. This clearly shows that the presence of a metal post will result in scatter and noise that is detrimental for the subjective image quality of CBCT scans. Moreover, the scatter and blooming caused by endodontic filling materials may mask cracks, narrow canals, and resorption lesions that are present on the treated or the adjacent teeth in clinical reality.

The type of tooth was a significant influencing factor on VAS score. This could be related to the number of root canals in the respective teeth. Anterior teeth contain fewer canals than premolars, which subsequently contain fewer canals than molars [29]. It was reasonable for observers to give a lower score for CBCT images that exhibit a rather complex root canal system with multiple canals, relative to a system with only one or two canals.

A recent survey has revealed that half of the endodontists in the USA had a CBCT device on-site [30]. Among the various indications, more than $50 \%$ of endodontists used CBCT imaging ("occasionally," "frequently," or "always") to detect missing canals. A similar survey from the UK also pointed out that assessment of complex root canal systems was one of the most important indications for the use of CBCT [31]. For both countries, CBCT images prescribed by endodontists most frequently involved small FOVs [30, 31]. These findings highlight the importance of the current evaluation on the effects of different exposure protocols (ULD, SD, and HR) on the image quality of CBCT images with small FOVs.
A relevant limitation of the current study was its in vitro setting. The teeth specimens were scanned without scatter and absorption from bone or skull materials or metallic crowns on adjacent teeth which might have influenced reconstruction and artefact expression, and no motion artifact was involved. The soft tissue simulated by silicone looked similar to the reported tissue simulation with water, which also focused on the subjective image quality of CBCT images [32]. Therefore, the results were obtained under relatively ideal circumstances, and will most likely result in less favorable ratings in a clinical scenario. These findings have to be interpreted with some caution, and need to be tested and validated under clinical conditions. Besides, the study only tested the low-dose protocol from one single CBCT device, as the present investigation was focused on the effect of specialists' different backgrounds rather than the differences between various $\mathrm{CBCT}$ devices. Moreover, there are certainly alternative methodologies to assess and compare subjective image quality of CBCT scans, such as the recognition or linear measurement of detectable anatomical structures or features.

Overall, the present data does not support the indiscriminate use of standard or high-resolution CBCT imaging for endodontic evaluations prior to and after root canal treatment. Overall, CBCT should ideally be reserved for specific diagnostic questions such as complex clinical situations and complications especially when $2 \mathrm{D}$ imaging and/or the surgical microscope are deemed inadequate. The current results demonstrate that the acquisition mode is less significant for the subjective image quality of $\mathrm{CBCT}$ scans than the observer specialty, stage of treatment (including the type of post used), and type of tooth involved. Further research is needed to evaluate the potential of low-dose CBCT protocols for endodontics, especially as the effective dose for a CBCT with a small FOV and a low-dose mode could be as low as in the range of $1-2$ panoramic views $[12,33,34]$. As there are many CBCT devices on the market, the present findings may at least partially be also dependent on device specifications, and thus cannot be directly extrapolated to other devices not tested in this study.

\section{Conclusions}

From the findings of the present in vitro study, the following conclusions can be drawn:

- A routine use of standard or high-resolution mode settings for CBCT images for assessing root canal systems prior to or following root canal treatment should be questioned.

- Observer specialty, stage of treatment, type of post, and type of tooth were all factors influencing subjective image quality of CBCT scans. 
- A low-dose CBCT mode for diagnostic purposes prior to or following root canal treatment results in favorable ratings regarding subjective image quality.

- As the data from the present study are based on an in vitro model distant from clinical situations, the findings need to be validated in future clinical investigations, ideally also using different $\mathrm{CBCT}$ devices.

Acknowledgments This study was partially supported by a Seed Fund for Basic Research from The University of Hong Kong (ref.-number: 201704159008). The authors thank Mr Shadow Kim Wai Yeung, Centralized Research Laboratory, Faculty of Dentistry, The University of Hong Kong, for his statistical assistance.

Funding Information Open access funding provided by University of Basel. This study was partially supported by a Seed Fund for Basic Research from The University of Hong Kong (ref.-number: 201704159008).

\section{Compliance with ethical standards}

Conflict of interest The authors declare that they have no conflict of interest.

Ethical approval All procedures performed were in accordance with the ethical standards of the institutional and/or national research committee and with the 1964 Helsinki declaration and its later amendments or comparable ethical standards. The study protocol was submitted to and approved by the local institutional review board (IRB) of the University of Hong Kong / Hospital Authority Hong Kong West Cluster (approval number UW 17-206).

Informed consent For this type of study (retrospective study), formal consent is not required.

Open Access This article is licensed under a Creative Commons Attribution 4.0 International License, which permits use, sharing, adaptation, distribution and reproduction in any medium or format, as long as you give appropriate credit to the original author(s) and the source, provide a link to the Creative Commons licence, and indicate if changes were made. The images or other third party material in this article are included in the article's Creative Commons licence, unless indicated otherwise in a credit line to the material. If material is not included in the article's Creative Commons licence and your intended use is not permitted by statutory regulation or exceeds the permitted use, you will need to obtain permission directly from the copyright holder. To view a copy of this licence, visit http://creativecommons.org/licenses/by/4.0/.

\section{References}

1. Omer O, Al Shalabi R, Jennings M, Glennon J, Claffey N (2004) A comparison between clearing and radiographic techniques in the study of the root-canal anatomy of maxillary first and second molars. Int Endod J 37(5):291-296

2. Velvart P, Hecker H, Tillinger G (2001) Detection of the apical lesion and the mandibular canal in conventional radiography and computed tomography. Oral Surg Oral Med Oral Pathol Oral Radiol Endod 92(6):682-688
3. Tamse A, Fuss Z, Lustig J, Kaplavi J (1999) An evaluation of endodontically treated vertically fractured teeth. J Endod 25(7): 506-508

4. Kersten DD, Mines P, Sweet M (2008) Use of the microscope in endodontics: results of a questionnaire. J Endod 34(7):804-807

5. Patel S (2009) New dimensions in endodontic imaging: Part 2. Cone beam computed tomography. Int Endod J 42(6):463-475

6. Patel S, Durack C, Abella F, Roig M, Shemesh H, Lambrechts P, Lemberg K (2014) European Society of Endodontology position statement: the use of CBCT in endodontics. Int Endod J 47(6):502 504

7. Neelakantan P, Khan K, Ng GPH, Yip CY, Zhang C, Cheung GSP (2018) Does the orifice-directed dentin conservation access design debride pulp chamber and mesial root canal systems of mandibular molars similar to a traditional access design? J Endod 44(2):274 279

8. Connert T, Zehnder MS, Weiger R, Kühl S, Krastl G (2017) Microguided endodontics: accuracy of a miniaturized technique for apically extended access cavity preparation in anterior teeth. J Endod 43(5):787-790

9. Jaju PP, Jaju SP (2015) Cone-beam computed tomography: Time to move from ALARA to ALADA. Imaging Sci Dent 45(4):263-265

10. Yeung AWK, Jacobs R, Bornstein MM (2019) Novel low-dose protocols using cone beam computed tomography in dental medicine: a review focusing on indications, limitations, and future possibilities. Clin Oral Investig 23(6):2573-2581

11. Ludlow JB, Ivanovic M (2008) Comparative dosimetry of dental CBCT devices and 64-slice CT for oral and maxillofacial radiology. Oral Surg Oral Med Oral Pathol Oral Radiol Endod 106(1):106114

12. Harris D, Horner K, Gröndahl K, Jacobs R, Helmrot E, Benic GI, Bornstein MM, Dawood A, Quirynen M (2012) EAO guidelines for the use of diagnostic imaging in implant dentistry 2011. A consensus workshop organized by the European Association for Osseointegration at the Medical University of Warsaw. Clin Oral Implants Res 23(11):1243-1253

13. Hidalgo Rivas JA, Horner K, Thiruvenkatachari B, Davies J, Theodorakou C (2015) Development of a low-dose protocol for cone beam CT examinations of the anterior maxilla in children. Br J Radiol 88(1054):20150559

14. Neelakantan P, Subbarao C, Ahuja R, Subbarao CV, Gutmann JL (2010) Cone-beam computed tomography study of root and canal morphology of maxillary first and second molars in an Indian population. J Endod 36(10):1622-1627

15. Michetti J, Maret D, Mallet J-P, Diemer F (2010) Validation of cone beam computed tomography as a tool to explore root canal anatomy. J Endod 36(7):1187-1190

16. Shelley A, Ferrero A, Brunton P, Goodwin M, Horner K (2015) The impact of CBCT imaging when placing dental implants in the anterior edentulous mandible: a before-after study. Dentomaxillofac Radiol 44(4):20140316

17. Koo TK, Li MY (2016) A guideline of selecting and reporting intraclass correlation coefficients for reliability research. J Chiropr Med 15(2):155-163

18. Costa FF, Pinheiro LR, Umetsubo OS, dos Santos JO, Gaia BF, Cavalcanti MGP (2014) Influence of cone-beam computed tomographic scan mode for detection of horizontal root fracture. J Endod 40(9):1472-1476

19. Neves FS, Freitas DQ, Campos PSF, Ekestubbe A, Lofthag-Hansen S (2014) Evaluation of cone-beam computed tomography in the diagnosis of vertical root fractures: the influence of imaging modes and root canal materials. J Endod 40(10):1530-1536

20. Li G (2013) Patient radiation dose and protection from cone-beam computed tomography. Imaging Sci Dent 43(2):63-69

21. Demirturk Kocasarac H, Helvacioglu Yigit D, Bechara B, Sinanoglu A, Noujeim M (2016) Contrast-to-noise ratio with 
different settings in a CBCT machine in presence of different rootend filling materials: an in vitro study. Dentomaxillofac Radiol 45(5):20160012

22. Ozcan G, Sekerci AE, Cantekin K, Aydinbelge M, Dogan S (2016) Evaluation of root canal morphology of human primary molars by using $\mathrm{CBCT}$ and comprehensive review of the literature. Acta Odontol Scand 74(4):250-258

23. Rodríguez G, Abella F, Durán-Sindreu F, Patel S, Roig M (2017) Influence of cone-beam computed tomography in clinical decision making among specialists. J Endod 43(2):194-199

24. McCaul L, McHugh S, Saunders W (2001) The influence of specialty training and experience on decision making in endodontic diagnosis and treatment planning. Int Endod J 34(8):594-606

25. Raitz R, Correa L, Curi M, Dib L, Fenyo-Pereira M (2006) Conventional and indirect digital radiographic interpretation of oral unilocular radiolucent lesions. Dentomaxillofac Radiol 35(3):165169

26. Kruse C, Spin-Neto R, Evar Kraft D, Væth M, Kirkevang LL (2019) Diagnostic accuracy of cone beam computed tomography used for assessment of apical periodontitis: an ex vivo histopathological study on human cadavers. Int Endod J 52(4):439-450

27. Soğur E, Baksı B, Gröndahl HG (2007) Imaging of root canal fillings: a comparison of subjective image quality between limited cone-beam CT, storage phosphor and film radiography. Int Endod J 40(3):179-185

28. Costa FF, Gaia BF, Umetsubo OS, Cavalcanti MGP (2011) Detection of horizontal root fracture with small-volume cone-beam computed tomography in the presence and absence of intracanal metallic post. J Endod 37(10):1456-1459
29. Neelakantan P, Subbarao C, Subbarao CV (2010) Comparative evaluation of modified canal staining and clearing technique, cone-beam computed tomography, peripheral quantitative computed tomography, spiral computed tomography, and plain and contrast medium-enhanced digital radiography in studying root canal morphology. J Endod 36(9):1547-1551

30. Setzer FC, Hinckley N, Kohli MR, Karabucak B (2017) A survey of cone-beam computed tomographic use among endodontic practitioners in the United States. J Endod 43(5):699-704

31. Rajeevan M, Chandler NP, Makdissi J, San Chong B (2018) A survey of cone beam computed tomography (CBCT) use among endodontic practitioners in the UK. Endodontic Pract Today 12(1): 29-33

32. Shelley AM, Brunton P, Horner K (2011) Subjective image quality assessment of cross sectional imaging methods for the symphyseal region of the mandible prior to dental implant placement. J Dent 39(11):764-770

33. Benavides E, Rios HF, Ganz SD, An C-H, Resnik R, Reardon GT, Feldman SJ, Mah JK, Hatcher D, Kim M-J (2012) Use of cone beam computed tomography in implant dentistry: the International Congress of Oral Implantologists consensus report. Implant Dent 21(2):78-86

34. Bornstein MM, Scarfe WC, Vaughn VM, Jacobs R (2014) Cone beam computed tomography in implant dentistry: a systematic review focusing on guidelines, indications, and radiation dose risks. Int J Oral Maxillofac Implants (29 (Suppl.)):55-77

Publisher's note Springer Nature remains neutral with regard to jurisdictional claims in published maps and institutional affiliations. 